\title{
"Assessment of Oral Hygiene Habits and Oral Health Awareness among Pre-University Students (16-18yrs) Of Mangalore"- A Questionnaire Study
}

\author{
Dr. Arun Kumar MS ${ }^{1}$, Dr. Rahmath Irshana ${ }^{2}$, Dr. Shashikanth Hegde ${ }^{3}$, \\ Dr.Rajesh $\mathrm{KS}^{4}$
}

\begin{abstract}
Purpose/ objective Health is a highly individualized concept, its perception is very much colored by individuals experience and cultural background. In parallel with the changing oral disease patterns there should be significant improvements in oral health awareness, dental knowledge and attitudes of children and parents as well. The present study was carried out to assess oral hygiene habits, oral health awareness and attitude among pre university students.

Methods A cross sectional study of 400 students from different Pre university colleges was conducted using a specially formulated objective type of questionnaire consisting of 24 questions with both open and close ended questions. The filled responses were statistically analysed using Frequency percentage and chi- square test. Results 56\% of the subjects were scared of dental treatment, among them $67.47 \%$ feared Injection, 59.8\% of the subjects have visited the dentist among them 51.9\% have been for routine dental check up. Most subjects brushed their teeth twice a day using tooth paste. However $78.3 \%$ of the subjects were not aware of the presence of fluoride in their tooth paste. About $62.2 \%$ of the subjects brushed for 2-5 minutes. $83.8 \%$ of the subjects agreed that smoking harms oral health and only $58.3 \%$ of the subjects attended free dental camps.

Conclusion Continuous implementation of school health education programs play a major role in educating the younger generation and also inculcating in them the better oral health practices. Oral health education can be included in the curriculum for children to emphasize a positive attitude towards oral health

Keywords: Oral health, Community Awareness, Health education.
\end{abstract}

\section{Introduction}

Health is a highly individualized concept, its perception is very much colored by individuals experience and cultural background. The world wide prevalence of dental diseases is a constant reminder for the universal need for effective dental health education programmes. The ways to improve oral health are complex but may involve a more sensible approach to sugar consumption, improved oral hygiene practices, fluorides in toothpaste, topical fluoride application, effective use of oral health services and establishment of school-based preventive programmes. In parallel with the changing oral disease patterns there should be significant improvements in oral health awareness, dental knowledge and attitudes of children and parents as well.

It is observed that certain oral diseases in adults can be controlled by changing the routine oral habits which may be reflected as a benefit of health education during individual's life cycle. An important task of oral health professionals is to instill in their patients the correct oral hygiene habits to prevent various oral diseases. Imparting knowledge to the young budding generation plays a key role in bringing awareness in the society.

Since there is lack of sufficient data pertaining to 16-18 yrs age group, the present study focuses on comprehensive overview of the oral health behaviour, knowledge and attitude towards oral hygiene among the pre university students.

\section{Objectives of The Study}

1. To assess oral hygiene habits among pre university students

2. To evaluate oral health awareness and attitude among pre university students

\section{Materials And Methods}

There is no universally accepted or recommended index/ inventory to measure dental health attitude and behavior. The data was collected on the attitude and behavioral aspects, derived from a series of independent questionnaires. The Hiroshima University- Dental Behavioral Inventory (HU-DBI) questionnaire developed by Kawamura has been demonstrated to be useful for assessing patient's perceptions and oral health behavior and is widely used all around. ${ }^{1}$

For the present study, a custom made questionnaire was prepared to explore the dental health attitudes, knowledge and behavior of pre university students of Mangalore. The questionnaire closely resembled the Hiroshima University- Dental Behavioral Inventory (HU-DBI) of Kawamura, but our questionnaire was 
formulated to derive more information with regards to the habit and attitude of the subjects pertaining to the regions. The data obtained may also help in assessing and comparing the focus on curriculum on preventive aspects, so as to decide whether pre university students should be introduced to practice of preventive health care.

\section{Study Population}

A cross sectional study of 400 students from different Pre university colleges situated in Mangalore city, Dakshina kannada District, Karnataka was conducted using a specially formulated objective type of questionnaire consisting of open and close ended questions.

Participation in the survey was voluntary and anonymity was maintained about the personal \& academic record. The respondents were asked to indicate only their gender. The questionnaire consisted of 24 questions and alloted time was 15 minutes. The filled responses were then transferred to the Microsoft excel sheet and statistically analysed using Frequency percentage and chi- square test.

\section{Results}

Different age groups and genders demonstrated no significant differences in their responses, so the frequency tables presented the subjects as a whole.

\section{Attitude}

Results reported that only $36.8 \%$ of the subjects were worried about their tooth colour. $56 \%$ of the subjects were scared of dental treatment, among them $67.47 \%$ feared Injection, $9.4 \%$ had fear of bleeding during the procedure and $9.8 \%$ of the subjects were scared of cross-infections in dental clinics.Among the total study population, $59.8 \%$ of the subjects have visited the dentist among them $51.9 \%$ have been for routine dental check up. It was observed that $26.8 \%$ of the subjects visited dentist once in three months. Observations also showed that $58.3 \%$ of the subjects attended free dental camps. (Graph-1)

\section{Oral hygiene Practices}

Most subjects brushed their teeth twice a day. About $62.2 \%$ of the subjects brushed for 2-5 minutes. It was noticed that tooth paste was used by all the subjects, while $33.5 \%$ of the subjects used mouth wash in addition to brushing in similar fashion as regards to frequency and duration of brushing. ( Graph -2)

\section{Awareness}

$71 \%$ of the subjects replaced the tooth brush once in three months. $32.8 \%$ had bleeding gums, among them $66.4 \%$ noticed it while brushing. $78.3 \%$ of the subjects were not aware of the presence of fluoride in their tooth paste. $83.8 \%$ of the subjects agreed that smoking harms oral health. (graph -3$)$

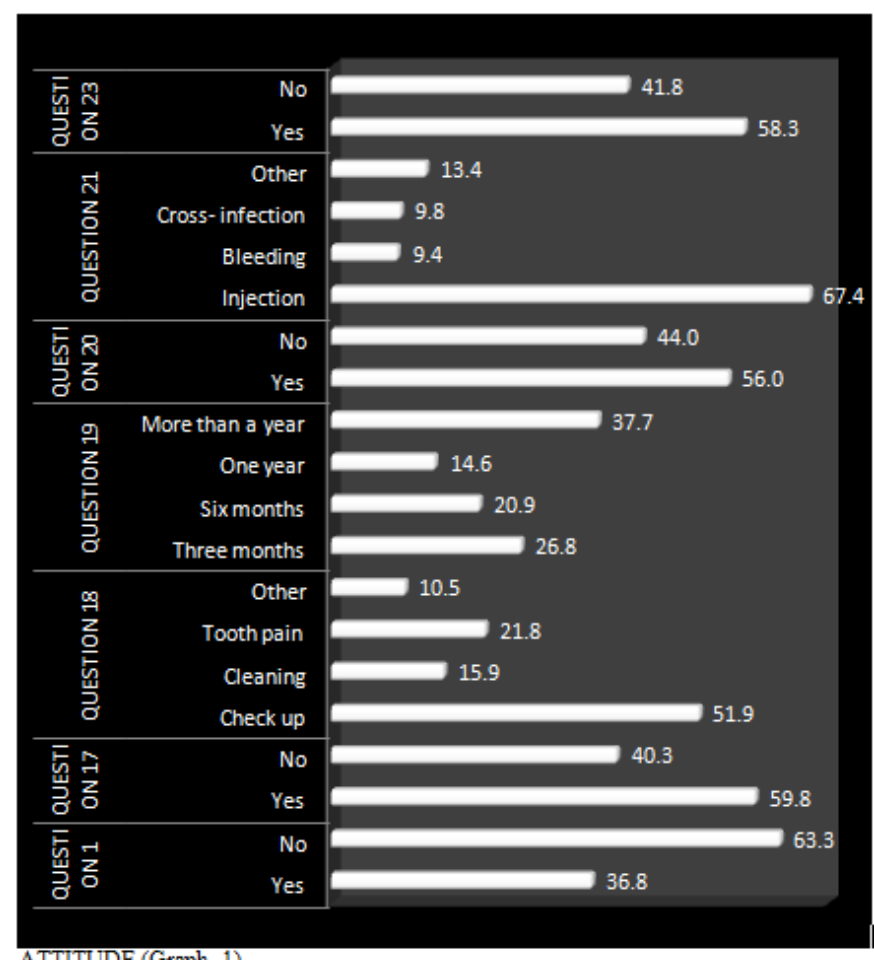

ATTITUDE (Graph -1) 


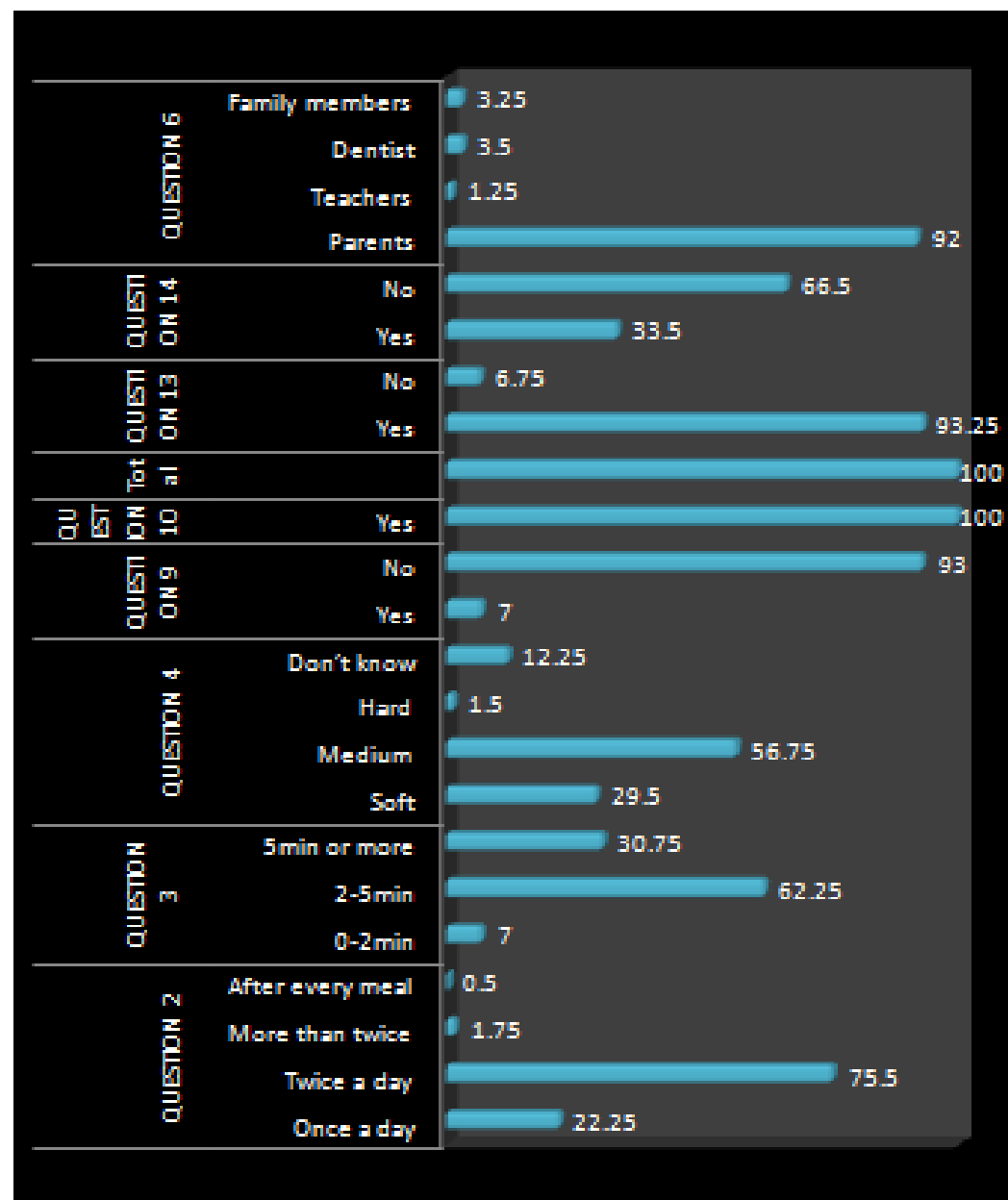

ORAI HYGIENE HABITS (GRAPH -2)

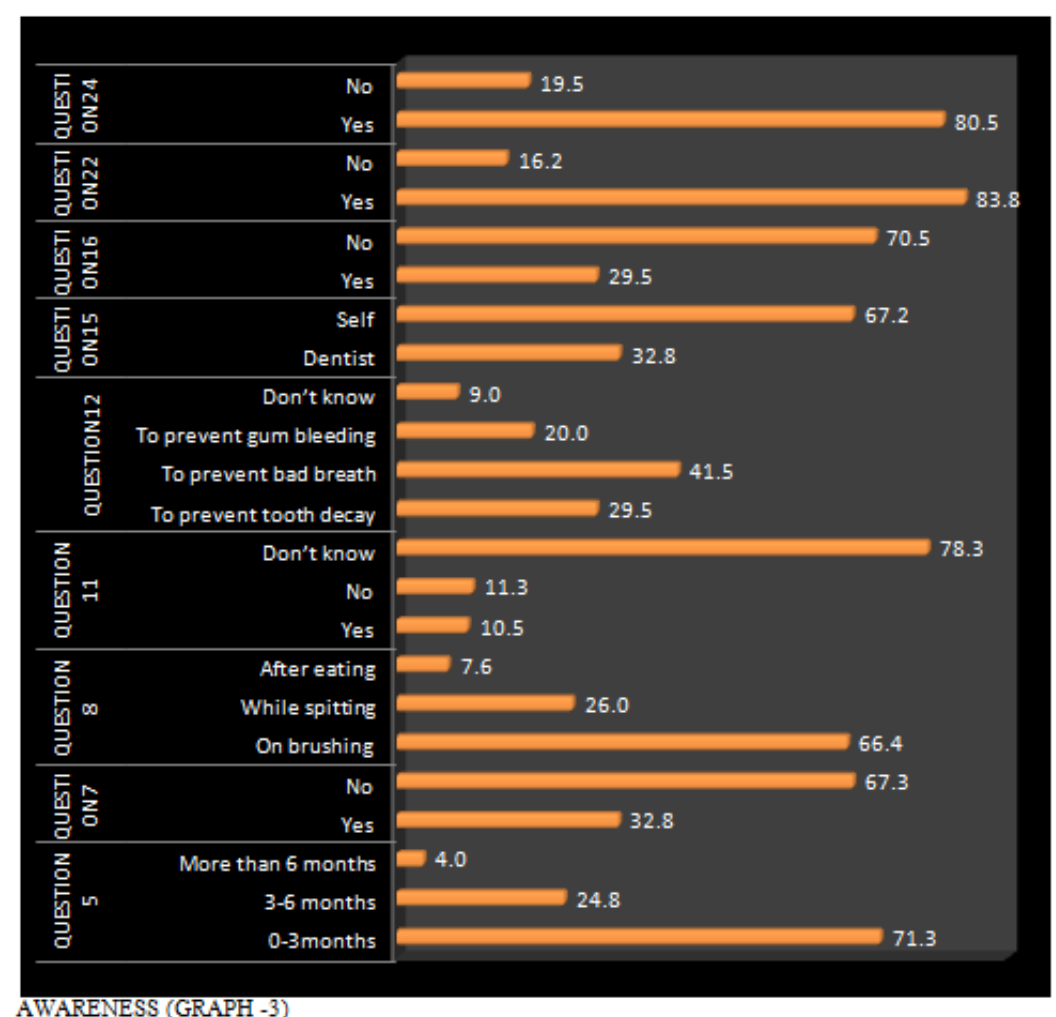




\section{Discussion}

The past fifty years has witnessed a reduction in the severity and prevalence of oral disease among the population of the developed countries ${ }^{2}$. The present study has provided a comprehensive overview of the oral health practices, knowledge, and attitudes of pre university students of age sixteen to eighteen studying in three colleges of Mangalore.

\section{Attitude}

One of the most frequent reasons for seeking dental treatment is anterior tooth discoloration ${ }^{3}$. Appearance of the dentition and colour of the teeth is of particular cosmetic importance among the larger population ${ }^{4}$. However did not seem to be a matter of major concern among the subjects in our study.

It was noticed that nearly $40.3 \%$ of the subjects had not visited dentist, major reason being the fear of dental treatment. Injection was the major fear factor among many. Sophisticated dental settings and instruments added to their anxiety levels. Fear of drilling was reported as a major hurdle for seeking dental treatment in a study by Mirza et al ${ }^{5}$.

Priya et $\mathrm{al}^{6}$ noted that fear among many was restricted to the first dental visit, however during the subsequent visits patients would become more cooperative. Nicolas et $\mathrm{al}^{7}$ reported that frequent exposure to dental experiences might be a positive factor in helping to reduce patient's anxiety levels. This emphasizes the fact that first dental appointment is crucial in developing a positive attitude towards dental treatment. The dentist's approach, behaviour and clinical surroundings influence the patient attitude. Previous studies among Jordanians showed that approximately 80 percent of Jordanian adults and children received dental examinations and treatment on an irregular basis and visited the dentist only for emergencies ${ }^{\mathbf{8}}$.

Missing awareness about the crucial role of regular dental checkups in detecting as well as preventing dental diseases is another gap in public education. As dentists its our responsibility to educate people and get patients motivated to visit dentist.

\section{Oral Hygiene Practices}

It was observed in our study that all 400 subjects used tooth brush and tooth paste to brush their teeth. This could probably reflect the awareness or affordability for tooth brush and paste among the study population. A study by Mahesh et al ${ }^{9}$ carried out in Chennai reported that children resorted to use of charcoal as a medium to brush their teeth than the tooth brush.

In the present study majority of the participants performed the recommended practice of brushing twice a day which is similar when compared with western industrialized countries ${ }^{\mathbf{1 0 1 1}}$, indicating a better oral hygiene practices among the study population. Study reported that the role of parents was significant in adopting appropriate brushing techniques. This emphasizes on the need for systematic community oriented oral health promotion programs for a better health.

The information on type of tooth brush used by the subjects showed that approximately $60 \%$ of them used recommended type of brush. However a small percentage of subjects were not aware of the type of tooth brush used. Usage of other oral hygiene aids such as dental floss and mouthwash was found to be limited. Similar results have been reported by Priya et $\mathrm{al}^{6}$. This could be probably due to inadequate knowledge transfer from dentist to patients or lack of public health education programs. This emphasizes the need for educating and getting the public motivated to use efficient oral hygiene measures for better oral health .

\section{Awareness}

In the present study, approximately $72 \%$ of the subjects replaced their tooth brush once in three months. The ADA recommends that consumers replace toothbrushes approximately every 3-4 months or sooner if the bristles become frayed with use. This illustrates the importance of regular replacement of tooth brushes and its role in maintaining oral health. A study by Ling et al ${ }^{12}$ reported less than $50 \%$ of the subjects replaced within three months.

Priya et $\mathrm{al}^{6}$ noticed that majority of children were not aware about bleeding gums and consequences of dental plaque. Present study also showed that $33 \%$ of subjects reported with bleeding gums among them two third of the subjects noticed it while brushing. This brings into fore the importance of self reporting by the patients, since bleeding gums is an initial sign of gingival disease, which can be used as a tool to educate people regarding the relation between bleeding gums and gingival health. In a study by Ling et $\mathrm{al}^{9} 80 \%$ of subjects of age group 18 yrs reported with bleeding gums. This indicates of a better oral awareness among the study subjects and reflects better gingival health.

It was noted that approximately $79 \%$ of study population were not aware of presence of fluoride in tooth paste but $29.5 \%$ were aware that fluoride prevented tooth decay.

Twetman et $\mathrm{al}^{13}$ reported a strong evidence of caries preventive effect of daily use of fluoride toothpaste, but awareness regarding the role of fluoride was lacking among the study population. 
However majority of subjects were aware of the fact that smoking harms oral health. Thus teaching adolescents about the negative oral health consequences of smoking alone may not be enough to prevent them from smoking. Preventive efforts in addition to enhancing skills to resist peer influence should also be carried out.

\section{Conclusion}

Community outreach programs, dental camps and wide availability of resources will probably serve as the best measure to increase oral awareness among the younger population. The use of other recommended oral hygiene methods such as dental floss and mouthwash was found to be rare; this also could be attributed to the lack of oral health education and/or the cost of such aids. Thus continuous implementation of school health education programs play a major role in educating the younger generation and also inculcating in them the better oral health practices. Oral health education can be included in the curriculum for children to emphasize a positive attitude towards oral health.

\section{References}

[1] Dagli R, Tadakamadla S, Dhanni C, Kulkami S. Self reported dental health attitude and behavior of dental students in India. J Oral Sci 2008; 50(3): 267-72.

[2] Downer MC. The improving oral health of United Kingdom adults and prospects for future. Br Dent J. 1991; 23:154-8.

[3] Amarlal D, Rayen R, Muthu MS. Macroabrasion in Pediatric Dentistry. J Clin Pediatr Dent. 2006; 31(1 ):9-13.

[4] Watts A, Addy M. Tooth discolouration and staining: a review of the literature. Br Dent J. 2001;190: 309-16.

[5] Mirza BA, Syed A, Izhar F, Ali Khan A. Oral health attitudes, knowledge, and behavior amongst high and low socioeconomic school going children in Lahore, Pakistan. Pak Oral Dent J. 2011;31: 396-401.

[6] Priya M, Devdas K, Amarlal D, Venkatachalapathy A. Oral health attitudes, knowledge and practice among school children in Chennai, India. J Educ Ethics Dent. 2013; 3:26-33.

[7] Nicolas E, Bersadent M, Collado V, Carrasco P. Factors affecting dental fear in French children aged 5-12 years. Int J Paediatric Dent. 2010; 20: 366-73.

[8] Taani DQ. Periodontal awareness and knowledge and pattern of dental attendance among adults in Jordan. Int Dent J. 2002; 52:94-8.

[9] Mahesh Kumar P, Joseph T, Varma RB, Jayanthi M. Oral health status of 5years and 12years school going children in Chennai city - An epidemiological study. J.Indian Soc Pedod Prev Dent. 2005; 23:17-22.

[10] Petersen PE, Danila I, Samoila A. Oral health behavior, knowledge, and attitudes of children, mothers, and school teachers in Romania in 1993. Acta Odontol Scand 1995; 53:363-8.

[11] King A, Wold B, Tudor-Smith C, Harel Y. Dietary habits, dental care and body image. In: World Health Organisation. The Health of Youth: A Cross-National Survey. Copenhagen: WHO Regional Office for Europe; 1995. p. 39-55.

[12] Ling Zhu, Poul Erik, Petersen, Hong-Ying Wang, Jin-You Bian and Bo Xue Zhang. Oral health knowledge, attitudes and behaviour of children and adolescents in China. Int Dent J. 2003; 53: 289-98.

[13] Twetman S . Caries preventive effect of fluoride tooth paste- A systematic review. Acta Odontol Scand. 2003; 61(6): $347-55$. 